\title{
Hybrid Stability Checking Method for Synchronization of Chaotic Fractional-Order Systems
}

\author{
Seng-Kin Lao, ${ }^{1}$ Lap-Mou Tam, ${ }^{1,2}$ Hsien-Keng Chen, ${ }^{3}$ and Long-Jye Sheu ${ }^{4}$ \\ ${ }^{1}$ Department of Electromechanical Engineering, Faculty of Science and Technology, University of Macau, \\ Avenue Padre Tomás Pereira, Taipa 999078, Macau \\ ${ }^{2}$ Institute for the Development and Quality, Avenue Padre Tomás Pereira, Taipa 999078, Macau \\ ${ }^{3}$ Department of Mechanical Engineering, Hsiuping University of Science and Technology, 11 Gongye Road, \\ Dali District, Taichung 412-80, Taiwan \\ ${ }^{4}$ Department of Mechanical Engineering, Chung Hua University, Section 2, 707 WuFu Road, Hsinchu 30012, Taiwan \\ Correspondence should be addressed to Hsien-Keng Chen; kane@mail.hust.edu.tw
}

Received 24 October 2013; Accepted 28 February 2014; Published 3 April 2014

Academic Editor: Dumitru Baleanu

Copyright (c) 2014 Seng-Kin Lao et al. This is an open access article distributed under the Creative Commons Attribution License, which permits unrestricted use, distribution, and reproduction in any medium, provided the original work is properly cited.

\begin{abstract}
A hybrid stability checking method is proposed to verify the establishment of synchronization between two hyperchaotic systems. During the design stage of a synchronization scheme for chaotic fractional-order systems, a problem is sometimes encountered. In order to ensure the stability of the error signal between two fractional-order systems, the arguments of all eigenvalues of the Jacobian matrix of the erroneous system should be within a region defined in Matignon's theorem. Sometimes, the arguments depend on the state variables of the driving system, which makes it difficult to prove the stability. We propose a new and efficient hybrid method to verify the stability in this situation. The passivity-based control scheme for synchronization of two hyperchaotic fractional-order Chen-Lee systems is provided as an example. Theoretical analysis of the proposed method is validated by numerical simulation in time domain and examined in frequency domain via electronic circuits.
\end{abstract}

\section{Introduction}

Nonlinear systems may exhibit dynamical chaotic behavior. The study of chaos synchronization has received increasing attention due to its predicted potentials in technological applications in recent years. In 1983, Fujisaka and Yamada [1] first described the synchronization of chaotic signals. Some years later, Pecora and Carroll [2] synchronized two identical chaotic systems with different initial conditions. Subsequently, research activities of chaos synchronization have grown continuously.

Fractional calculus has been studied in a speedy pace during the recent years. It has been implemented in various engineering fields such as control [3], modeling [4], thermal engineering [5], and bioengineering [6]. There are mainly two ways to approximate a fractional-order system: in frequency domain based on Riemann-Liouville definition or GrunwaldLetnikov definition and in time domain based on Caputo definition. In frequency domain, linear approximation may be obtained with a given discrepancy over a frequency range [7]. The discrepancy and frequency range must be carefully chosen; otherwise, huge error may occur between the approximate and true results [8]. There are other frequency domain methods using continued fraction expansions and interpolation techniques [9], FIR filters [10], and so forth. Regrettably, the time memory characteristic of fractionalorder systems is not considered. Meanwhile, the approximation in time domain outperforms the frequency domain approximation. The improved $\mathrm{P}(\mathrm{EC})^{m} \mathrm{E}$ method based on the Adams-Bashforth-Moulton algorithm [11] produces pretty accurate results. The only drawback is its heavy computation load. Recently, discrete fractional calculus [12] starts drawing researchers' attention. For example, various versions of fractional discrete-time Logistic map have been proposed and studied using different approaches, such as left Caputo discrete delta difference [13], discontinuous dynamical systems [14], and discrete-time Fourier transform [15]. Meanwhile, other discrete fractional systems have also been developed 
[16]. Jarad et al. investigated the stability of discrete fractional systems in detail using the Lyapunov direct method [17]. It is also interesting to develop applications such as chaos synchronization in fractional discrete systems.

In 2004, Chen and Lee [18] developed a new chaotic system based on the Euler equations for the motion of a rigid body. This system describes the chaotic behavior for the anticontrol of chaos in rigid-body motion. The system is then called the Chen-Lee system [19]. Complete synchronization [20], synchronization and antisynchronization [21], controlling chaos with multiple time-delays [22], electronic circuit implementation $[23,24]$, fractional-order behavior $[19,25]$, and so forth have been studied for this system recently. It is believed that hyperchaotic systems are demanded by practical engineering applications, such as secure communication. The hyperchaotic Chen-Lee system and its hybrid projective synchronization were proposed by Chen et al. [26]. Due to more complex structure in the differential equations of the hyperchaotic Chen-Lee system, the synchronization was achieved under a complicate controller. Furthermore, the hyperchaotic Chen-Lee system may exhibit even more complex dynamical behavior in the fractional-order domain. It is hence necessary to seek for a simple and effective controller for those systems.

The concept of passive control theory [27] has been a focus for the control of chaos and chaos synchronization. A simple state-feedback controller has been built based on the passivity to stabilize the Lorenz equation [28] and Rabinovich system [29]. Chaos synchronization in the unified chaotic system, the Rikitake attractor, and the hyperchaotic complex Chen system with unknown parameters has been achieved using the passive control technique [30-32]. Passivity synchronization has been implemented to fractionalorder hyperchaotic Liu's systems [33] by using inequality relation through absolute maximum bound of its system variable. Unfortunately, it is not always that the stability can be determined by upper bounds of system variables. This happens to the case when we apply passivity theory to the hyperchaotic Chen-Lee system. Hence, we proved the control scheme based on the proposed stability checking procedure without using any bound of system's variable.

In this paper, the synchronization between two hyperchaotic Chen-Lee systems with different initial conditions was established via the passive control technique first. The influence of the controller parameters was discussed for enhancing the efficiency of synchronization. Next, the proposed controller was also applied to fractional-order hyperchaotic Chen-Lee systems. Synchronization was ensured based on the stability theorem by Matignon [34]. An efficient and easy strategy was proposed to find the range of eigenvalues when the Jacobian matrix involves system states. Finally, numerical simulation and the corresponding electronic circuits were included to show the feasibility and effectiveness of the proposed methods.

\section{Preliminaries}

Matignon's theorem and the passivity theory were reviewed in this section. The former was used for analyzing the stability of fractional differential equations and the latter for designing the synchronization scheme between two systems.

2.1. The Matignon Theorem. Consider an error dynamical system $e(x, y, t)$, where $x(t)$ and $y(t)$ are the driving and response systems and $J_{0}(x)$ the Jacobian matrix which contains $x(t)$, and $q$ is the fractional order of the systems.

Theorem 1 (see [34]). The trivial solution of the system $e(x, y$, t) is asymptotically stable if and only if

$$
|\arg (\lambda)|>\frac{q \pi}{2}
$$

is satisfied for all eigenvalues $\lambda$ of Jacobian matrix $J_{0}$ of system $e(x, y, t)$ evaluated at the origin.

Corollary 2. The response system is asymptotically synchronized with the driving system if and only if $|\arg (\lambda)|>q \pi / 2$ is satisfied for all eigenvalues $\lambda(x, t)$ of $J_{0}(x)$ for any possible $x(t)$.

2.2. Review of the Passivity Theory. Consider the following nonlinear system [27]:

$$
\begin{gathered}
\dot{x}=f(x)+g(x) u, \\
y=h(x),
\end{gathered}
$$

where the state variable $x \in \mathfrak{R}^{m}$, the input $u \in \mathfrak{R}^{m}$, and the output $y \in \mathfrak{R}^{m} . f(x)$ and $g(x)$ are the smooth vector fields and $f(0)=0 \cdot h(x)$ is a smooth mapping. The system (2) is passive if the conditions below are all satisfied.

(1) $f(x)$ and $g(x)$ exist.

(2) For all $t \geq 0$, there is a real constant $\beta$ such that the inequality holds

$$
\int_{0}^{t} u^{T}(\tau) y(\tau) d \tau \geq \beta
$$

or there are a real constant $\beta$ and a constant $\rho>0$ such that

$$
\int_{0}^{t} u^{T}(\tau) y(\tau) d \tau+\beta \geq \int_{0}^{t} \rho y^{T}(\tau) y(\tau) d \tau .
$$

If a system is passive, a suitable controller can stabilize asymptotically the equilibrium point $x=0$ of system (2).

If the system (2) has the relative degree $\{1, \ldots, 1\}$ at $x=$ $0\left(L_{g} h(0)\right.$ is nonsingular) and the distribution spanned by the vector field $g_{1}(x), \ldots, g_{m}(x)$ is involutive, then it can be represented in the generalized form

$$
\begin{aligned}
& \dot{z}=f_{0}(z)+p(z, y) y, \\
& \dot{y}=b(z, y)+k(z, y) u,
\end{aligned}
$$

where $k(z, y)$ is nonsingular $\forall(z, y)$. By designing a suitable controller $u$, the system (5) may be passive. Thus, the system (5) can be asymptotically stabilized to the equilibrium point by applying the controller $u$. If the system (5) describes an error dynamical system, then the synchronization between two systems is achieved. 
Theorem 3. The system (5) is asymptotically stabilized to the equilibrium point by applying the controller as follows:

$$
u=k^{-1}(z, y)\left[-b^{T}(z, y)-\frac{\partial}{\partial z} W(z) p(z, y)-\alpha y+v\right]
$$

where $W(z)$ is the Lyapunov function of $f_{0}(z)$ and $W(0)=0, \alpha$ is a positive real constant, and $v$ is an external signal connected to the reference input.

\section{Chaos Synchronization of Hyperchaotic Chen-Lee Systems}

Chen et al. [26] introduced the hyperchaotic Chen-Lee system:

$$
\begin{aligned}
& \dot{x}_{1}=-x_{2} x_{3}+a x_{1}, \\
& \dot{x}_{2}=x_{1} x_{3}+b x_{2}, \\
& \dot{x}_{3}=\frac{1}{3} x_{1} x_{2}+c x_{3}+0.2 x_{4}, \\
& \dot{x}_{4}=0.5 x_{2} x_{3}+0.05 x_{4}+d x_{1},
\end{aligned}
$$

where $a=5, b=-10, c=-3.8$, and $d$ are system parameters. Hyperchaotic behaviors have been observed when $0 \leq d \leq$ 1.3 excluding $d=0.8$ and $d=1.1$. For the synchronization purpose, consider that the system (7) is the driving system and the response system is defined as

$$
\begin{aligned}
& \dot{y}_{1}=-y_{2} y_{3}+a y_{1}+u_{1}, \\
& \dot{y}_{2}=y_{1} y_{3}+b y_{2}, \\
& \dot{y}_{3}=\frac{1}{3} y_{1} y_{2}+c y_{3}+0.2 y_{4}+u_{2}, \\
& \dot{y}_{4}=0.5 y_{2} y_{3}+0.05 y_{4}+d y_{1}+u_{3},
\end{aligned}
$$

where $u_{1}, u_{2}$, and $u_{3}$ are the controllers to be designed. Let $e_{1}=y_{1}-x_{1}, e_{2}=y_{2}-x_{2}, e_{3}=y_{3}-x_{3}$, and $e_{4}=y_{4}-x_{4}$. The error dynamical system is then expressed by

$$
\begin{aligned}
& \dot{e}_{1}=a e_{1}-e_{2} e_{3}-e_{2} x_{3}-e_{3} x_{2}+u_{1}, \\
& \dot{e}_{2}=b e_{2}+e_{1} e_{3}+e_{1} x_{3}+e_{3} x_{1}, \\
& \dot{e}_{3}=c e_{3}+\frac{1}{3}\left(e_{1} e_{2}+e_{1} x_{2}+e_{2} x_{1}\right)+0.2 e_{4}+u_{2}, \\
& \dot{e}_{4}=0.5\left(e_{2} e_{3}+e_{2} x_{3}+e_{3} x_{2}\right)+0.05 e_{4}+d e_{1}+u_{3} .
\end{aligned}
$$

By choosing $u_{2}=-(4 / 3) e_{2} x_{1}-0.2 e_{4}$ and $u_{3}=-0.5\left(y_{2} y_{3}-\right.$ $\left.x_{2} x_{3}\right)-\sigma e_{4}$, where $\sigma \geq 0.05$, the system (9) can be rewritten as

$$
\begin{aligned}
& \dot{e}_{1}=a e_{1}-e_{2} e_{3}-e_{2} x_{3}-e_{3} x_{2}+u_{1}, \\
& \dot{e}_{2}=b e_{2}+e_{1} e_{3}+e_{1} x_{3}+e_{3} x_{1}, \\
& \dot{e}_{3}=c e_{3}+\frac{1}{3}\left(e_{1} e_{2}+e_{1} x_{2}-e_{2} x_{1}\right), \\
& \dot{e}_{4}=(0.05-\sigma) e_{4}+d e_{1} .
\end{aligned}
$$

The aim is to design the controller $u_{1}$ for stabilizing the system (10) at the origin asymptotically. Therefore, the driving and the response systems are synchronized globally asymptotically.

Let $z_{1}=e_{2}, z_{2}=e_{3}, z_{3}=e_{4}$, and $y=e_{1}$. The system (10) can be rewritten as

$$
\begin{aligned}
& \dot{z}_{1}=b z_{1}+x_{1} z_{2}+\left(z_{2}+x_{3}\right) y, \\
& \dot{z}_{2}=c z_{2}-x_{1} z_{1}+\frac{1}{3}\left(z_{1}+x_{2}\right) y, \\
& \dot{z}_{3}=(0.05-\sigma) z_{3}+d y \\
& \dot{y}=a y-z_{1} z_{2}-z_{1} x_{3}-z_{2} x_{2}+u_{1} .
\end{aligned}
$$

It is in the form of (5), where

$$
\begin{gathered}
f_{0}(z)=\left[\begin{array}{c}
b z_{1}+x_{1} z_{2} \\
c z_{2}-x_{1} z_{1} \\
0.05-\sigma
\end{array}\right], \quad p(x, y)=\left[\begin{array}{c}
z_{2}+x_{3} \\
\frac{1}{3}\left(z_{1}+x_{2}\right) \\
d
\end{array}\right], \\
b(z, y)=a y-z_{1} z_{2}-z_{1} x_{3}-z_{2} x_{2}, \quad k(z, y)=1 .
\end{gathered}
$$

Because $b<0, c<0$, and $\sigma \geq 0.05$,

$$
\frac{d}{d t} W(z)=\left[z_{1}, z_{2}, z_{3}\right]\left[\begin{array}{c}
b z_{1}+x_{1} z_{2} \\
c z_{2}-x_{1} z_{1} \\
(0.05-\sigma) z_{3}
\end{array}\right] \leq 0 .
$$

According to Theorem 3, the system (10) is stabilized asymptotically at the origin if we choose the controller $u_{1}$ as

$$
u_{1}=\frac{2}{3} e_{3} x_{2}-\frac{1}{3} e_{2} e_{3}-d e_{4}-(a+\alpha) e_{1}+\nu .
$$

This means that the driving system (7) and the response system (8) can be synchronized globally asymptotically via the designed controller.

If the ordinary differential operators in (7) are replaced by fractional differential ones, we have the fractional-order hyperchaotic Chen-Lee system as shown as follows:

$$
\begin{aligned}
& D^{q} x_{1}=-x_{2} x_{3}+a x_{1}, \\
& D^{q} x_{2}=x_{1} x_{3}+b x_{2}, \\
& D^{q} x_{3}=\frac{1}{3} x_{1} x_{2}+c x_{3}+0.2 x_{4}, \\
& D^{q} x_{4}=0.5 x_{2} x_{3}+0.05 x_{4}+d x_{1} .
\end{aligned}
$$

By taking (15) as the driving system and letting

$$
\begin{aligned}
& D^{q} y_{1}=-y_{2} y_{3}+a y_{1}+u_{1}, \\
& D^{q} y_{2}=y_{1} y_{3}+b y_{2}, \\
& D^{q} y_{3}=\frac{1}{3} y_{1} y_{2}+c y_{3}+0.2 y_{4}+u_{2}, \\
& D^{q} y_{4}=0.5 y_{2} y_{3}+0.05 y_{4}+d y_{1}+u_{3}
\end{aligned}
$$




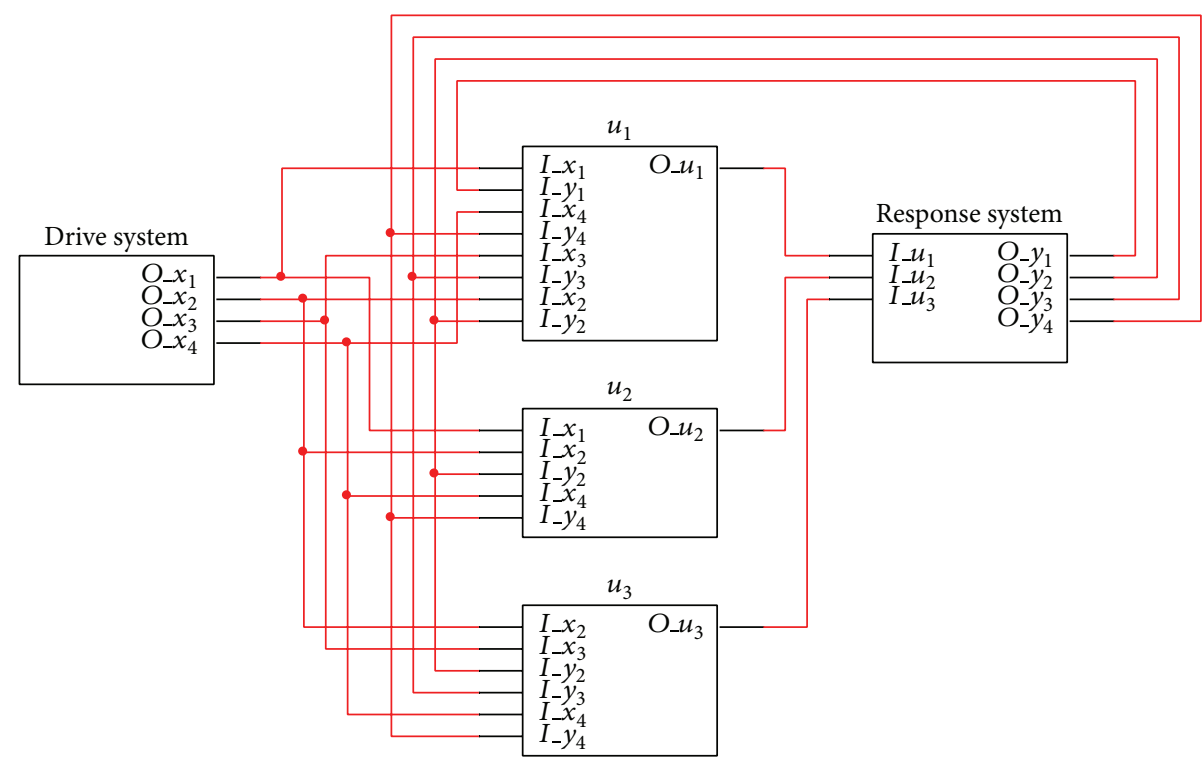

FIGURE 1: Schematic diagram of the synchronization system.

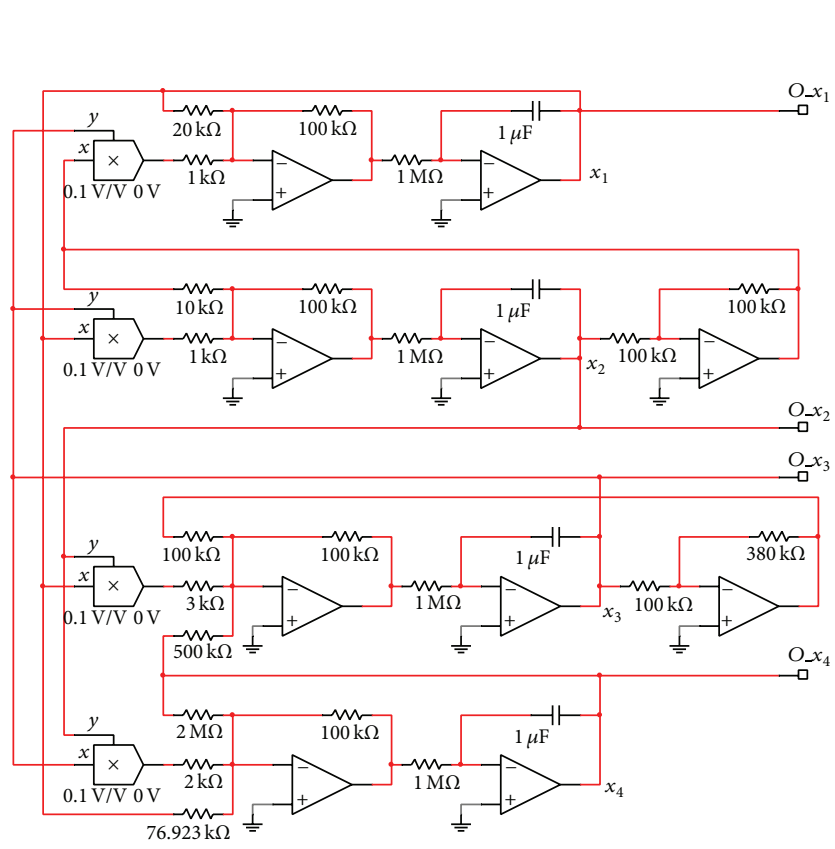

(a)

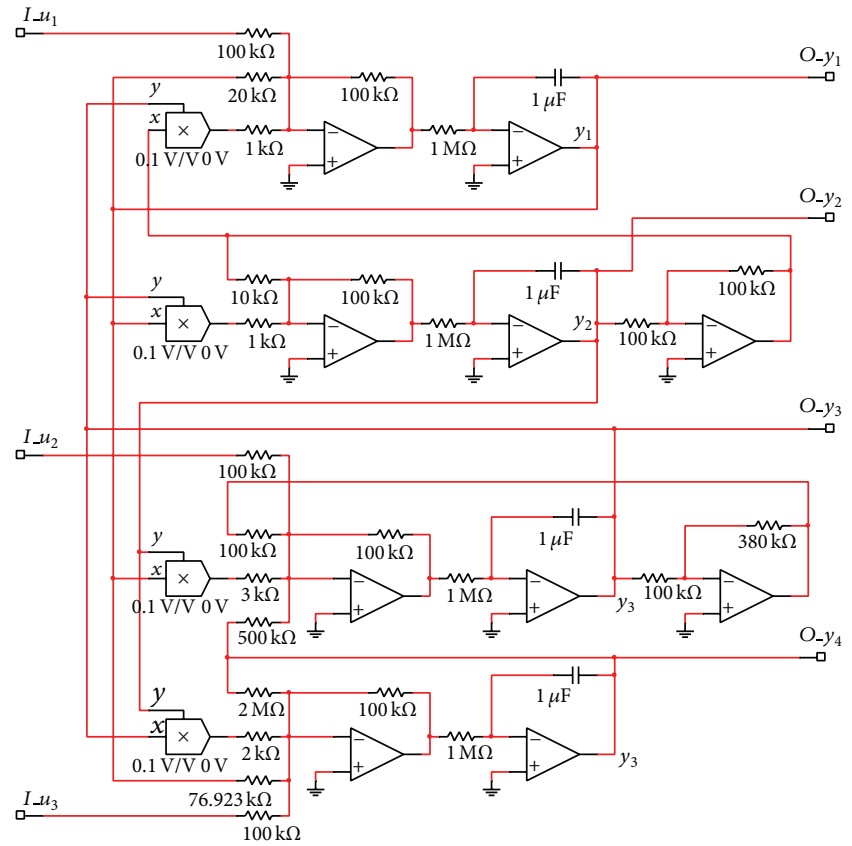

(b)

FIGURE 2: Electronic circuits of (a) the driving system and (b) the response system.

be the response system and supposing that the external source input $v=0$, the synchronization error between two fractional-order hyperchaotic Chen-Lee systems with identical order is then expressed as

$$
\begin{aligned}
& D^{q} e_{1}=-\alpha e_{1}-\frac{4}{3} e_{2} e_{3}-e_{2} x_{3}-\frac{1}{3} e_{3} x_{2}-d e_{4}, \\
& D^{q} e_{2}=b e_{2}+e_{1} e_{3}+e_{1} x_{3}+e_{3} x_{1},
\end{aligned}
$$

$$
\begin{aligned}
& D^{q} e_{3}=c e_{3}+\frac{1}{3}\left(e_{1} e_{2}+e_{1} x_{2}-e_{2} x_{1}\right), \\
& D^{q} e_{4}=(0.05-\sigma) e_{4}+d e_{1},
\end{aligned}
$$

where $q \in[0.98,1)$ is the fractional order. It has not been determined yet whether the passivity theory above can directly be applied to the synchronization for the fractional 


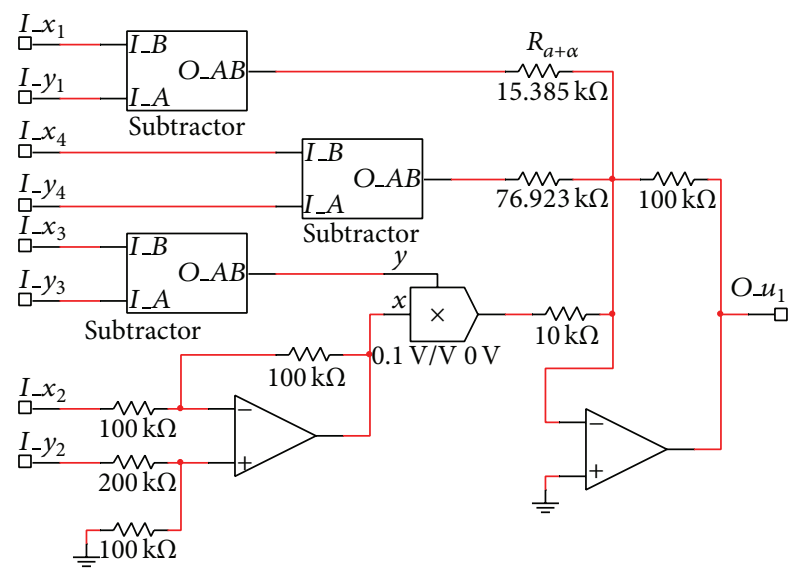

(a)

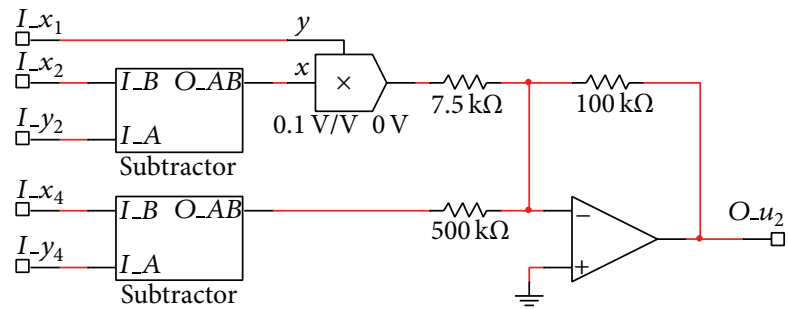

(b)

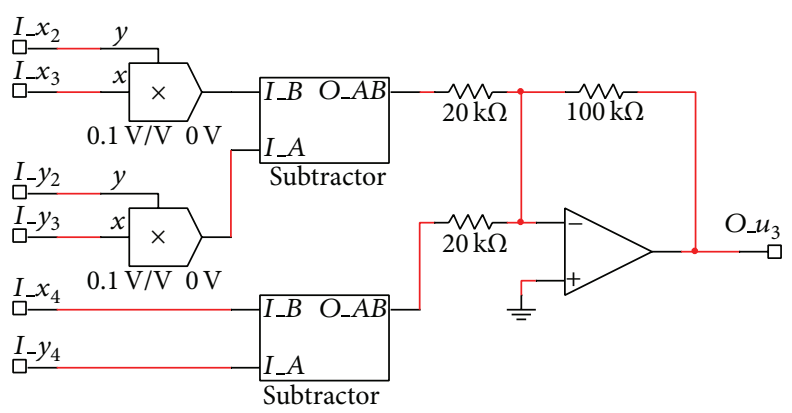

(c)

Figure 3: Electronic circuit of the controllers: (a) $u_{1}$; (b) $u_{2}$; (c) $u_{3}$.

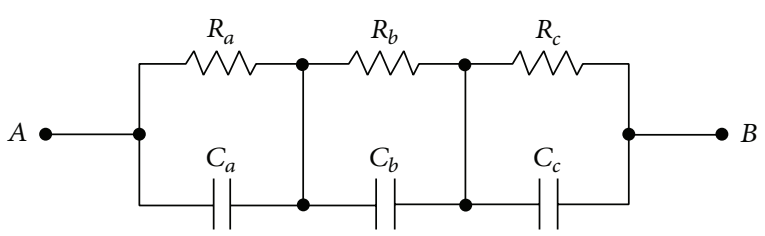

FIGURE 4: Chain fractance of $1 / s^{0.98}$.

counterpart or not. The stability of (17) can be determined by the following theorem.

Theorem 4. System (17) is globally asymptotically stable at the origin if $|\arg (\lambda(t))|>q \pi / 2$ is satisfied for all $\lambda(t)$ in

$$
\begin{aligned}
\lambda^{4}+ & \lambda^{3}(\alpha-b-c-\omega) \\
& +\lambda^{2}\left[\frac{1}{3} x_{1}^{2}+\frac{1}{9} x_{2}^{2}-x_{3}^{2}+d^{2}-\omega(\alpha-b-c)+\zeta\right] \\
& +\lambda\left[\frac{4}{9} x_{1} x_{2} x_{3}+\frac{1}{3}(\alpha-\omega) x_{1}^{2}-\frac{1}{9}(b+\omega) x_{2}^{2}+(c+\omega) x_{3}^{2}\right. \\
& \left.\quad-d^{2}(b+c)-\zeta \omega+\alpha b c\right] \\
& -\frac{4}{9} \omega x_{1} x_{2} x_{3}+\frac{1}{3}\left(d^{2}-\alpha \omega\right) x_{1}^{2}+\frac{1}{9} b \omega x_{2}^{2} \\
& -c \omega x_{3}^{2}+b c d^{2}-\alpha b c \omega=0
\end{aligned}
$$

for all sets of $x(t)=\left[x_{1}(t), x_{2}(t), x_{3}(t)\right]^{T}$ of system (15), where $\omega=0.05-\sigma$ and $\zeta=b c-\alpha(b+c)$.

Proof. To gain the eigenvalues at the origin, $e_{1}=e_{2}=e_{3}=$ $e_{4}=0$, we let

$$
\begin{aligned}
& \operatorname{det}\left|J_{0}(x)-\lambda I\right| \\
& =\operatorname{det}\left|\begin{array}{cccc}
-\alpha-\lambda & -x_{3} & -\frac{1}{3} x_{2} & -d \\
-x_{3} & b-\lambda & x_{1} & 0 \\
\frac{1}{3} x_{2} & -\frac{1}{3} x_{1} & c-\lambda & 0 \\
d & 0 & 0 & 0.05-\sigma-\lambda
\end{array}\right|=0
\end{aligned}
$$

and we have

$$
\begin{aligned}
\lambda^{4}+ & \lambda^{3}(\alpha-b-c-\omega) \\
+ & \lambda^{2}\left[\frac{1}{3} x_{1}^{2}+\frac{1}{9} x_{2}^{2}-x_{3}^{2}+d^{2}-\omega(\alpha-b-c)+\zeta\right] \\
+ & \lambda\left[\frac{4}{9} x_{1} x_{2} x_{3}+\frac{1}{3}(\alpha-\omega) x_{1}^{2}-\frac{1}{9}(b+\omega) x_{2}^{2}+(c+\omega) x_{3}^{2}\right. \\
& \left.-d^{2}(b+c)-\zeta \omega+\alpha b c\right]
\end{aligned}
$$



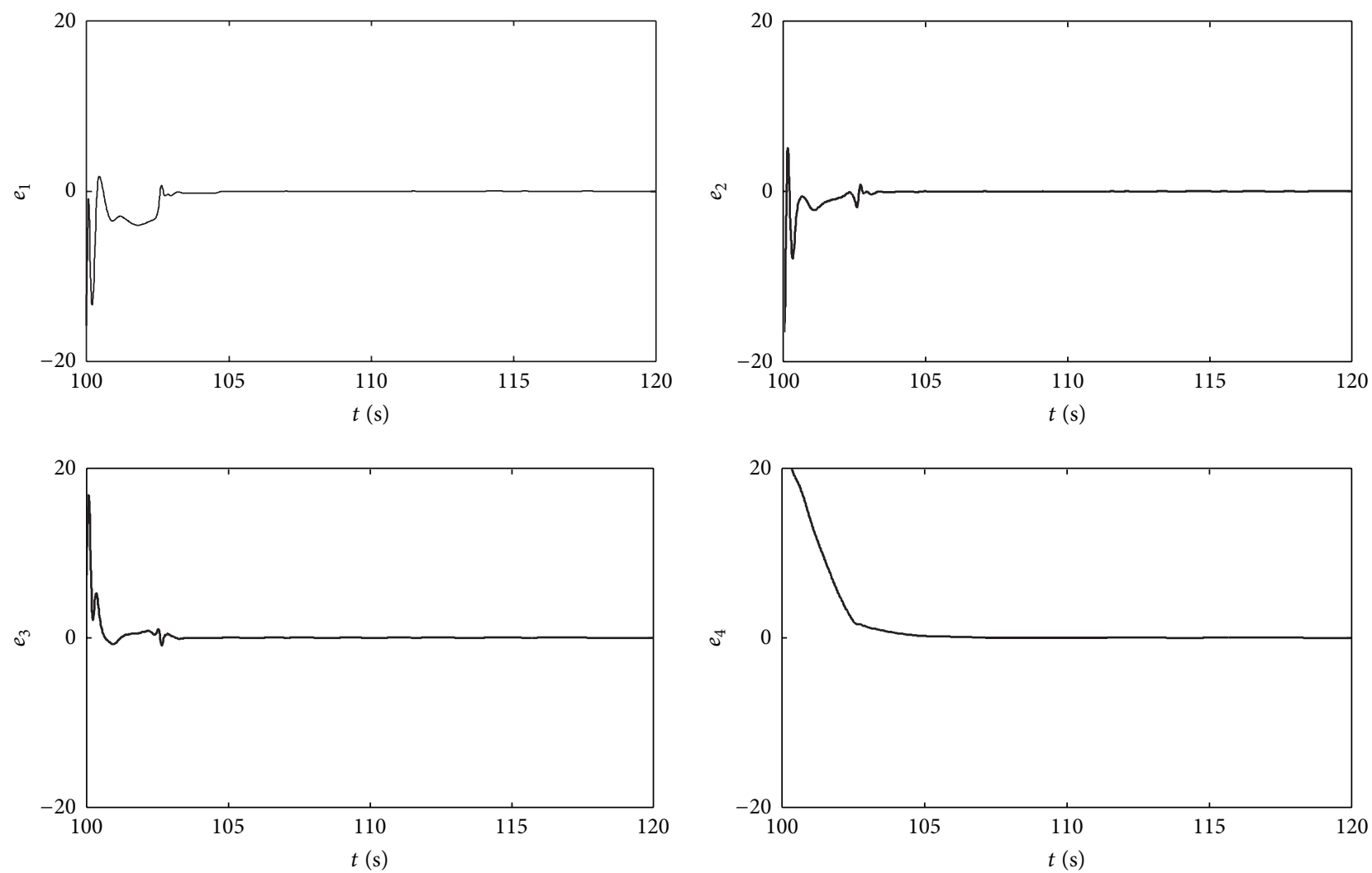

FIGURE 5: Time histories of the synchronization errors (10) between systems (7) and (8) with $\sigma=0.5$.
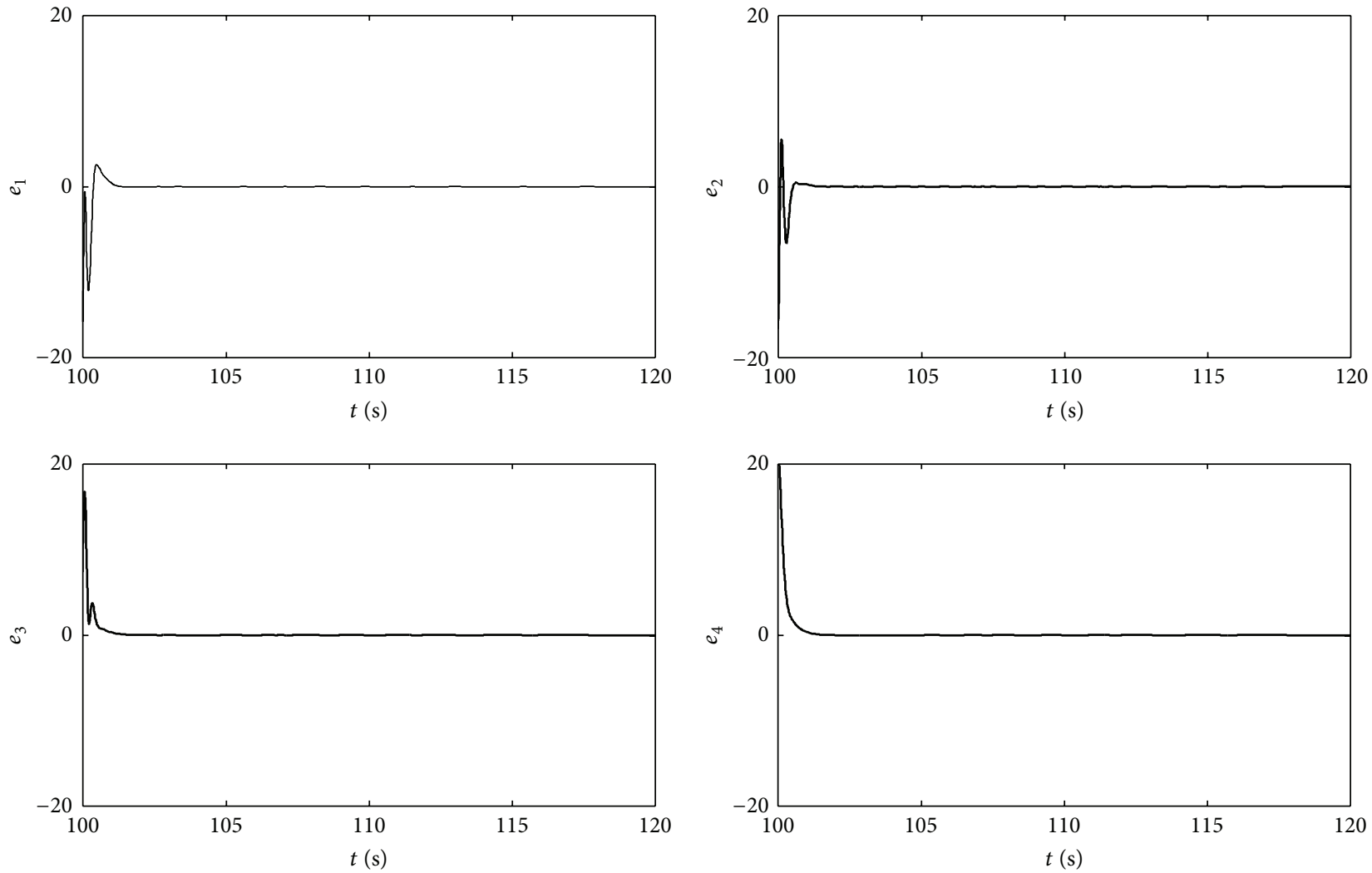

FIgURE 6: Time histories of the synchronization errors (10) between systems (7) and (8) with $\sigma=5$. 


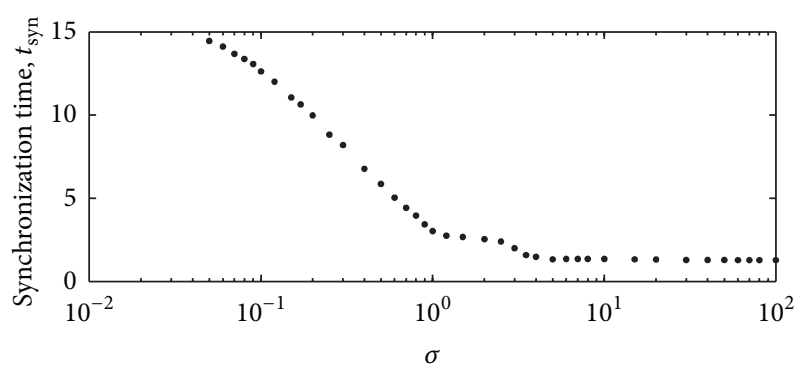

FIGURE 7: The influence of the controller parameter $\sigma$ on the synchronization time $t_{\text {syn }}$.

$$
\begin{aligned}
& -\frac{4}{9} \omega x_{1} x_{2} x_{3}+\frac{1}{3}\left(d^{2}-\alpha \omega\right) x_{1}^{2}+\frac{1}{9} b \omega x_{2}^{2} \\
& -c \omega x_{3}^{2}+b c d^{2}-\alpha b c \omega=0 .
\end{aligned}
$$

Obviously, the eigenvalues depend on the system states of (15). However, it is difficult to determine the stability by just considering the bounds of the system states. In order to examine the stability, we apply Theorem 1 . The procedure is described in the following. Time histories of the system states are first numerically evaluated for a sufficient long time, so that they can reflect the dynamical behavior of the chaotic fractional-order system. Next, the eigenvalues $\lambda(t)$ are evaluated for each set of the system states. The minimum values of $|\arg (\lambda(t))|$ are then collected at every time step. If all $\min |\arg (\lambda(t))|>q \pi / 2$, it implies that system (17) is stabilized at the origin. This completes the proof.

\section{Electronic Circuits}

An electronic circuit was constructed for implementing the proposed scheme. Figure 1 showed the synchronization system, which consisted of a driving system, a response system, and three controllers. The systems (7) and (8) were normalized by a factor of 10 . The controller parameter $\sigma$ was set to 5 . The corresponding electronic circuits of the systems and controllers were presented in Figures 2 and 3.

The above circuit can be converted to a fractional-order one by simply replacing the capacitors on the feedback path of the integrators by chain fractance [35]. Chain fractance is constructed of a group of resistors in parallel with a capacitor in series.

To approximately realize the fractional-order operator with $q=0.98$, the corresponding transfer function [7] with discrepancy of $0.5 \mathrm{~dB}$ between the actual and approximated signals over the frequency bandwidth $\omega_{\max }=100$ for a corner frequency $p_{T}=0.01$ was

$$
H(s) \approx \frac{1.2234 s^{2}+1463.2 s+4893.2}{(s+0.0106)(s+3.7716)(s+1341.4)} .
$$

It can be realized by a chain fractance of order 3 (Figure 4). Its transfer function was described by

$$
H(s)=\left(R_{a} / / \frac{1}{s C_{a}}\right)+\left(R_{b} / / \frac{1}{s C_{b}}\right)+\left(R_{c} / / \frac{1}{s C_{c}}\right) .
$$

The values of the resistors and capacitors of the chain fractance were then determined by solving the equations

$$
\begin{gathered}
\left(R_{a} C_{a}\right)^{-1}=0.0106, \\
\left(R_{b} C_{b}\right)^{-1}=3.7716, \\
\left(R_{c} C_{c}\right)^{-1}=1341.4, \\
C_{a}^{-1}+C_{b}^{-1}+C_{c}^{-1}=1.2234, \\
\frac{R_{a} C_{a}\left(R_{b}+R_{c}\right)+R_{b} C_{b}\left(R_{a}+R_{c}\right)+R_{c} C_{c}\left(R_{a}+R_{b}\right)}{R_{a} C_{a} R_{b} C_{b} R_{c} C_{c}}=1463.2, \\
\frac{R_{a}+R_{b}+R_{c}}{R_{a} C_{a} R_{b} C_{b} R_{c} C_{c}}=4893.2
\end{gathered}
$$

which gave $R_{a}=91.17 \mathrm{M} \Omega, R_{b}=32.046 \mathrm{k} \Omega, R_{c}=101.12 \Omega$, $C_{a}=1.0656 \mu \mathrm{F}, C_{b}=8.5245 \mu \mathrm{F}$, and $C_{c}=7.596 \mu \mathrm{F}$.

\section{Numerical Results}

In this section, the Runge-Kutta method of order 4 was used to solve the differential equations in the systems (7) and (8), while the improved method [11] based on Caputo derivative was implemented to approximate the fractional differential equations in (15) and (16) with time step size $\Delta t=0.001 \mathrm{~s}$. The control scheme took place at $100 \mathrm{~s}$ in all cases.

5.1. Synchronization in Integer-Order Systems. First of all, the value of $d$ was chosen to be $d=1.3$, with the fact that the Lyapunov exponents of the system (7) were $\lambda_{1}=0.677$, $\lambda_{2}=0.107, \lambda_{3}=0$, and $\lambda_{4}=-13.412 \lambda_{4}=-13.412$. Two positive Lyapunov exponents indicated the system (7) is hyperchaotic [26]. Next, the control parameters were chosen to be $\sigma=0.5, \alpha=1.5$, and $\nu=0$. The initial conditions for the driving and response systems were chosen as $[2,3,2,2]^{T}$ and $[4,-6,-6,-3]^{T}$, respectively. Figure 5 showed the time histories of the synchronization errors between the two systems (7) and (8). The response system was synchronized with the driving system in $5.9 \mathrm{~s}$. The synchronization time was shortened by setting $\sigma=5$. The results in Figure 6 showed that synchronization was well achieved within $1.1 \mathrm{~s}$. In addition, Figure 7 represented the influence of $\sigma$ on the synchronization time. It was noticed that the performance of synchronization was improved by increasing $\sigma$ from a small value up to $\sigma=5$. If $\sigma>5$, the synchronization time only slightly decreased with increasing $\sigma$, though the influence was quite unnoticeable.

5.2. Synchronization in Fractional-Order Systems. The order of the systems was chosen to be $q=0.98$. The phase diagrams of the system (15) were plotted in Figure 8(a). A four-scroll attractor was found in the $x-y$ plot, which made the system (15) exhibit more complicated dynamical behavior than the system (7). The stability of the error dynamical system (17) was examined by Theorem 4 . When $\alpha=5$ and $\sigma=5$, the time history of $\min |\arg (\lambda(t))|$ was plotted in Figure 9(a). 

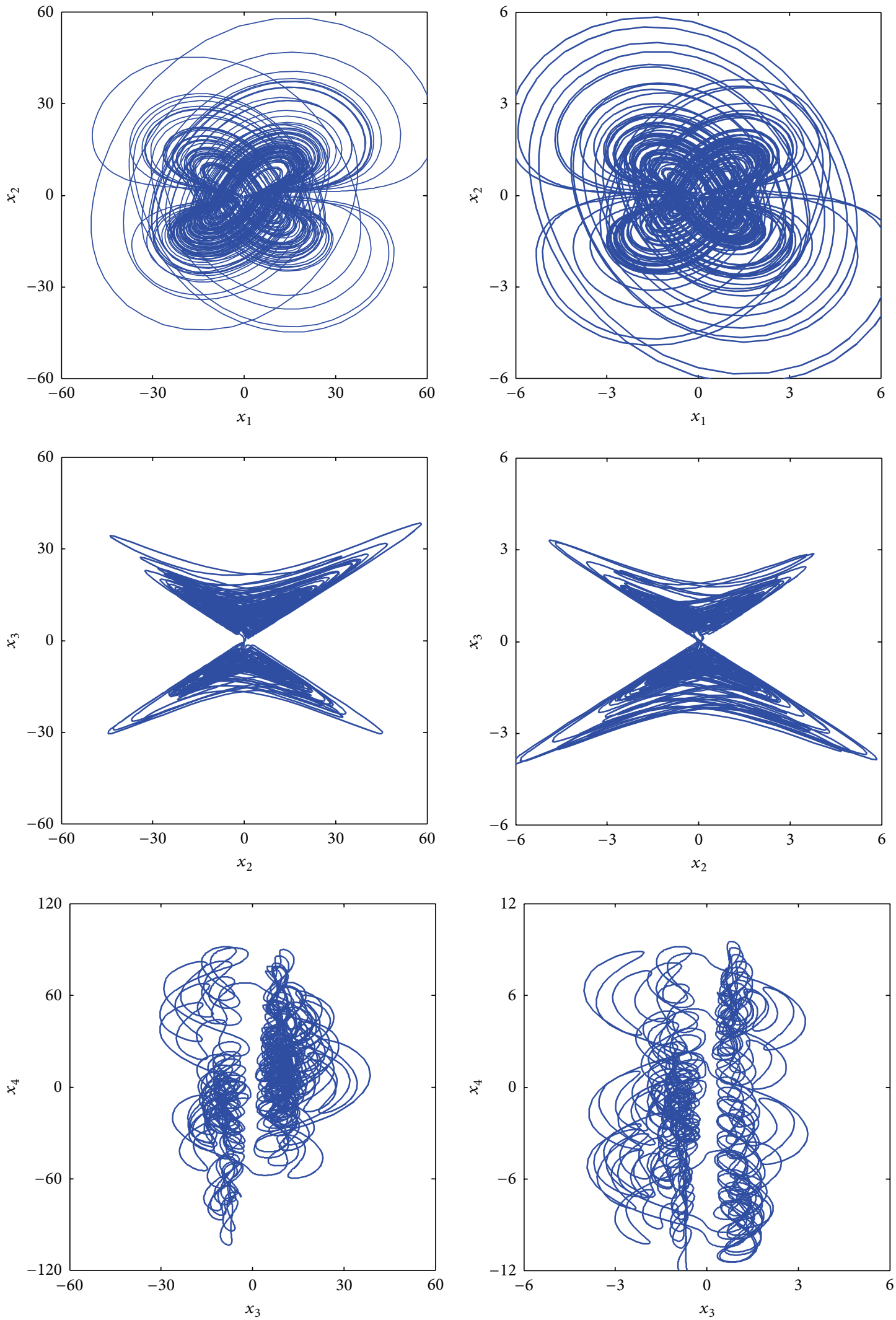

(a)

(b)

FIGURE 8: Phase diagrams of the hyperchaotic systems: (a) numerical solution in time domain; (b) circuit simulation in frequency domain. 


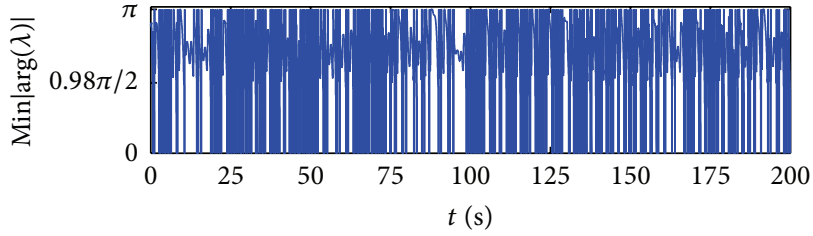

(a)

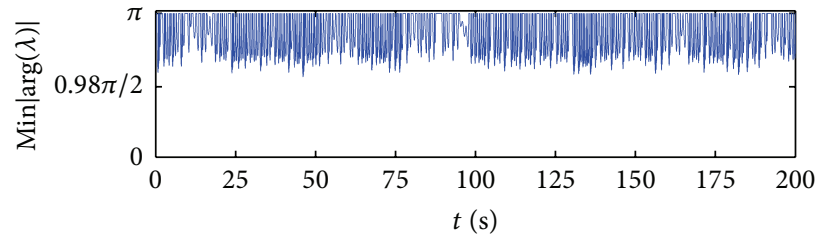

(b)

Figure 9: Time histories of $\min |\arg (\lambda(t))|:$ (a) $\alpha=5$; (b) $\alpha=155$.
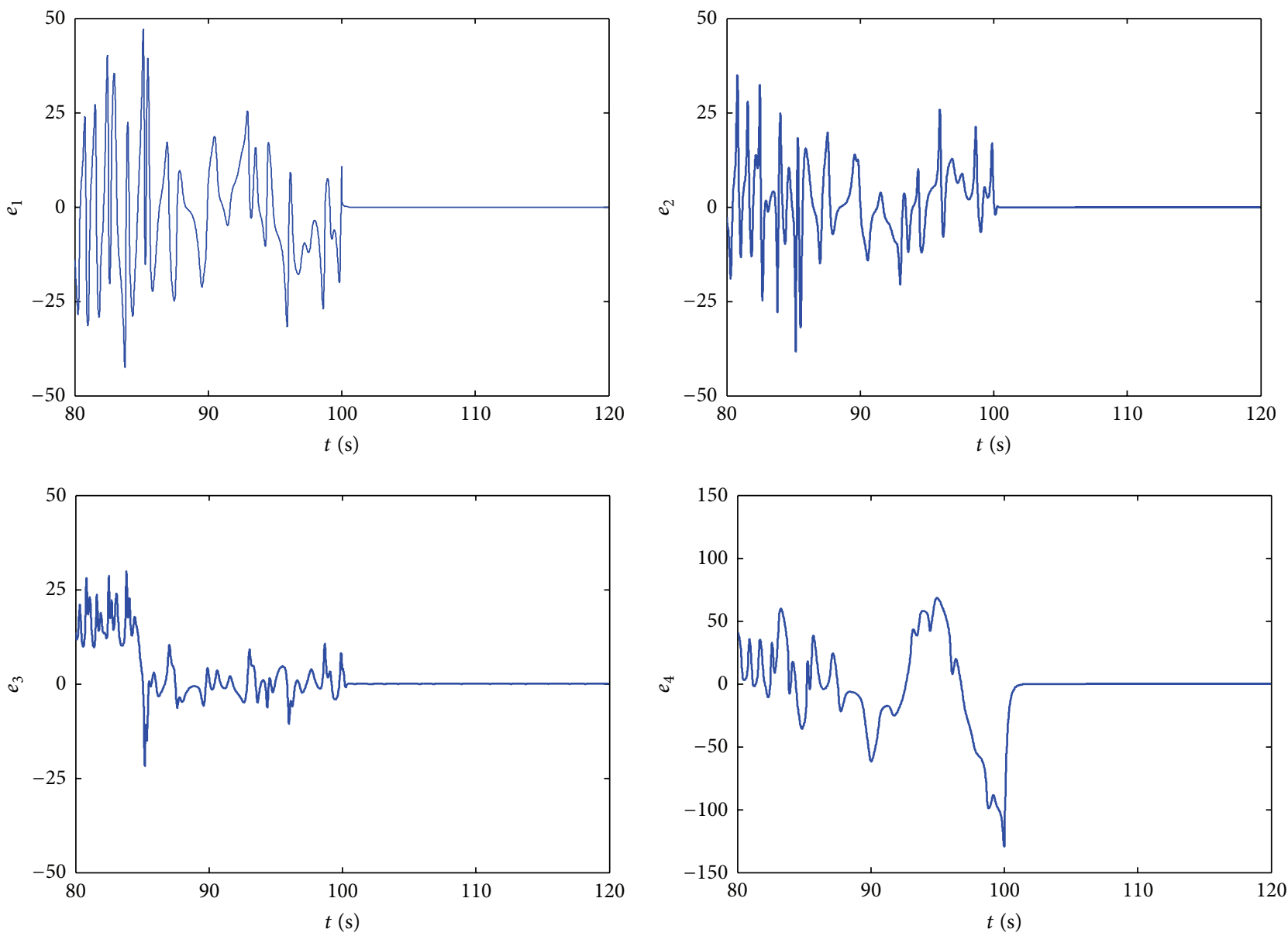

FIGURE 10: Time histories of the error dynamical system (17) when $\alpha=155$.

Obviously min $|\arg (\lambda(t))|$ reached zero at some $t$. According to Theorem 1, this implied that the synchronization may not be achieved. All values of $\min |\arg (\lambda(t))|$ were completely greater than $0.98 \pi / 2$ until $\alpha$ increased to 155 , as depicted in Figure 9(b). Under this condition, the response system (16) was synchronized successfully with the driving system (15). The time histories of the error system (17) were plotted in Figure 10. After the synchronization scheme took place at $t=100 \mathrm{~s}$, the error signal was asymptotically stabilized at the origin, meaning that the synchronization was well achieved.

5.3. Circuit Simulations. The simulation was done on Multisim package. The initial conditions were set to
$[0.2,0.3,0.2,0.2]^{T}$ and $[0.4,-0.6,-0.6,-0.3]^{T}$ for the integerorder driving and response systems, respectively. The outputs of the circuit simulation were depicted in Figure 11. The driving and response systems developed differently during the time period $0<t<100 \mathrm{~s}$. The response system was synchronized with the driving system after activating the controller at $t=100 \mathrm{~s}$. It meant that the synchronization circuit with integer order was built successfully.

For simulating systems with fractional order, the capacitors were replaced by chain fractance. The value of the resistor $R_{a+\alpha}=15.385 \mathrm{k} \Omega$ in Figure 3(a) was changed to $650 \Omega$ for $\alpha=155$. The numerical circuit-simulated phase diagrams of the hyperchaotic Chen-Lee system with order $q=0.98$ were depicted in Figure 8(b). The output 

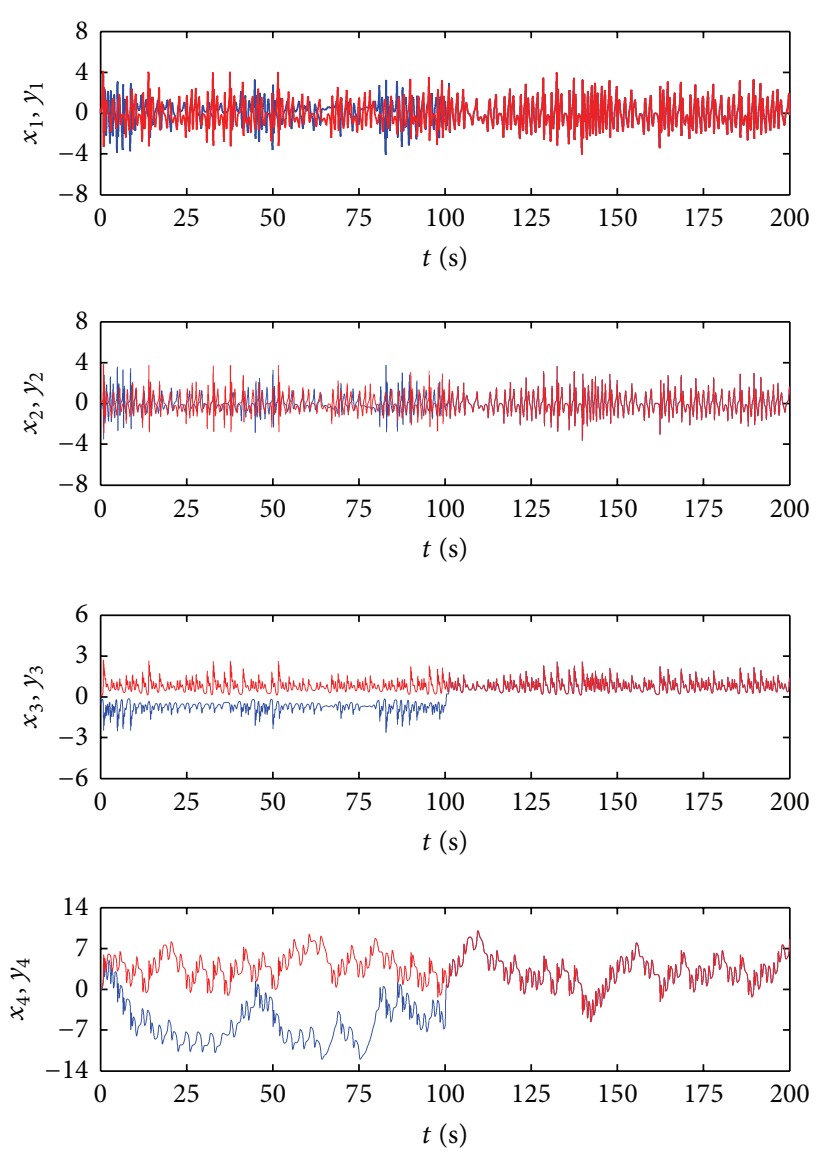

FIGURE 11: Time histories of the circuit outputs of the driving system (red) and the response system (blue) with integer order.

trajectories of the circuit simulation were plotted in Figure 12. It was clear that the response system was well synchronized with the driving system after $100 \mathrm{~s}$. Both circuit simulations were in good agreement with their corresponding numerical results.

\section{Conclusions}

A novel and efficient strategy was proposed to examine the stability of the error dynamical systems with fractional order. The chaos synchronization between two hyperchaotic ChenLee systems with fractional order was achieved via feedback passive control technique. The passive controller was first designed for the integer-order system. The control scheme was proved based on the stability theorem for fractional calculus. Numerical simulation was given to validate the proposed approaches. Chain fractance was designed for approximating the fractional order $q=0.98$ and the electronic circuits were simulated to verify the scheme. It is discovered that the synchronization scheme designed for integer-order systems may not directly be valid for systems with fractional order. Fortunately, the scheme may become effective by just simply changing a single parameter.
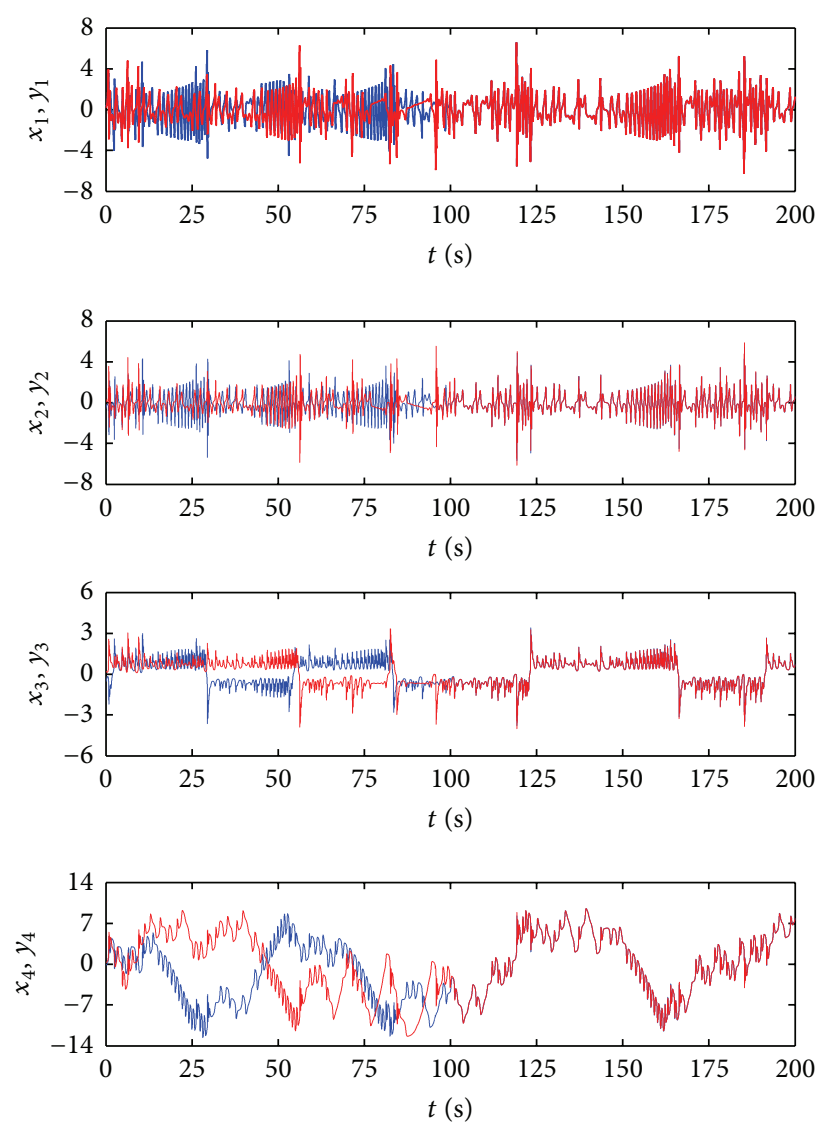

FIGURE 12: Time histories of the circuit outputs of the driving system (red) and the response system (blue) with fractional order.

\section{Conflict of Interests}

The authors declare that there is no conflict of interests regarding the publication of this paper.

\section{Acknowledgments}

The authors would like to thank the referees for their valuable suggestions and comments. This research was partially supported by a Grant (NSC98-2212-M-164-001-MY3) from the National Science Council, Taiwan.

\section{References}

[1] H. Fujisaka and T. Yamada, "Stability theory of synchronized motion in coupled-oscillator systems," Progress of Theoretical Physics, vol. 69, no. 1, pp. 32-47, 1983.

[2] L. M. Pecora and T. L. Carroll, "Synchronization in chaotic systems," Physical Review Letters, vol. 64, no. 8, pp. 821-824, 1990.

[3] A. G. Butkovskii, S. S. Postnov, and E. A. Postnova, "Fractional integro-differential calculus and its control-theoretical applications. II. Fractional dynamic systems: modeling and hardware implementation," Automation and Remote Control, vol. 74, no. 5, pp. 725-749, 2013. 
[4] M. Berli, "Challenges in the application of fractional derivative models in capturing solute transport in porous media: darcyscale fractional dispersion and the influence of medium properties," Mathematical Problems in Engineering, vol. 2013, Article ID 878097, 10 pages, 2013.

[5] X. J. Yang and D. Baleanu, "Fractional heat conduction problem solved by local fractional variation iteration method," Thermal Science, vol. 17, no. 5, pp. 625-628, 2013.

[6] R. L. Magin, "Fractional calculus models of complex dynamics in biological tissues," Computers \& Mathematics with Applications, vol. 59, no. 5, pp. 1586-1593, 2010.

[7] A. Charef, H. H. Sun, Y.-Y. Tsao, and B. Onaral, "Fractal system as represented by singularity function," Institute of Electrical and Electronics Engineers. Transactions on Automatic Control, vol. 37, no. 9, pp. 1465-1470, 1992.

[8] M. S. Tavazoei and M. Haeri, "Limitations of frequency domain approximation for detecting chaos in fractional order systems," Nonlinear Analysis. Theory, Methods \& Applications Series A: Theory and Methods, vol. 69, no. 4, pp. 1299-1320, 2008.

[9] K. Matsuda and H. Fujii, " $H_{\infty}$ optimized wave-absorbing control: analytical and experimental results," Journal of Guidance, Control, and Dynamics, vol. 16, no. 6, pp. 1146-1153, 1993.

[10] I. Petráš, "A note on the fractional-order Chua's system," Chaos, Solitons and Fractals, vol. 38, no. 1, pp. 140-147, 2008.

[11] K. Diethelm, "Efficient solution of multi-term fractional differential equations using $\mathrm{P}(\mathrm{EC})^{m}$ E methods," Computing. Archives for Scientific Computing, vol. 71, no. 4, pp. 305-319, 2003.

[12] K. S. Miller and B. Ross, "Fractional difference calculus," in Proceedings of the International Symposium on Univalent Functions, Fractional Calculus and Their Applications, pp. 139152, Nihon University, Koriyama, Japan, 1989.

[13] G.-C. Wu and D. Baleanu, "Discrete fractional logistic map and its chaos," Nonlinear Dynamics. An International Journal of Nonlinear Dynamics and Chaos in Engineering Systems, vol. 75, no. 1-2, pp. 283-287, 2014.

[14] A. M. A. El-Sayed and M. E. Nasr, "Discontinuous dynamical systems and fractional-orders difference equations," Journal of Fractional Calculus and Applications, vol. 4, no. 1, pp. 130-138, 2013.

[15] H. Zhao, H. K. Kwan, and J. Yu, "Fractional discrete-time chaotic map," in Proceedings of IEEE International Symposium on Circuits and Systems (ISCAS '06), pp. 21-24, May 2006.

[16] G. C. Wu, D. Baleanu, and S.-D. Zeng, "Discrete chaos in fractional sine and standard maps," Physics Letters A, vol. 378, no. 5-6, pp. 484-487, 2014.

[17] F. Jarad, T. Abdeljawad, D. Baleanu, and K. Biçen, "On the stability of some discrete fractional nonautonomous systems," Abstract and Applied Analysis, vol. 2012, Article ID 476581, 9 pages, 2012.

[18] H. K. Chen and C. I. Lee, "Anti-control of chaos in rigid body motion," Chaos, Solitons and Fractals, vol. 21, no. 4, pp. 957-965, 2004.

[19] L. M. Tam and W. M. Si Tou, "Parametric study of the fractionalorder Chen-Lee system," Chaos, Solitons and Fractals, vol. 37, no. 3, pp. 817-826, 2008.

[20] L. J. Sheu, H. K. Chen, J. H. Chen et al., "Complete synchronization of two Chen-Lee systems," Journal of Physics: Conference Series, vol. 96, no. 1, Article ID 012138, 2008.

[21] J. H. Chen, H. K. Chen, and Y. K. Lin, "Synchronization and anti-synchronization coexist in Chen-Lee chaotic systems," Chaos, Solitons and Fractals, vol. 39, no. 2, pp. 707-716, 2009.
[22] J. H. Chen, "Controlling chaos and chaotification in the ChenLee system by multiple time delays," Chaos, Solitons and Fractals, vol. 36, no. 4, pp. 843-852, 2008.

[23] L. J. Sheu, L. M. Tam, H. K. Chen, and S. K. Lao, "Alternative implementation of the chaotic Chen-Lee system," Chaos, Solitons and Fractals, vol. 41, no. 4, pp. 1923-1929, 2009.

[24] L. M. Tam, S. K. Lao, H. K. Chen, and L. J. Sheu, "Hybrid projective synchronization for the fractional-order Chen-Lee system and its circuit realization," Applied Mechanics and Materials, vol. 300, pp. 1573-1578, 2013.

[25] H.-K. Chen, L.-J. Sheu, L.-M. Tam, and S.-K. Lao, "A new finding of the existence of Feigenbaum's constants in the fractional-order Chen-Lee system," Nonlinear Dynamics. An International Journal of Nonlinear Dynamics and Chaos in Engineering Systems, vol. 68, no. 4, pp. 589-599, 2012.

[26] C.-H. Chen, L.-J. Sheu, H.-K. Chen et al., "A new hyper-chaotic system and its synchronization," Nonlinear Analysis. Real World Applications. An International Multidisciplinary Journal, vol. 10, no. 4, pp. 2088-2096, 2009.

[27] C. I. Byrnes, A. Isidori, and J. C. Willems, "Passivity, feedback equivalence, and the global stabilization of minimum phase nonlinear systems," Institute of Electrical and Electronics Engineers. Transactions on Automatic Control, vol. 36, no. 11, pp. 1228-1240, 1991.

[28] W. Yu, "Passive equivalence of chaos in Lorenz system," IEEE Transactions on Circuits and Systems I: Fundamental Theory and Applications, vol. 46, no. 7, pp. 876-878, 1999.

[29] S. Emiroçlu and Y. Uyaroğlu, "Control of Rabinovich chaotic system based on passive control," Scientific Research and Essays, vol. 5, no. 21, pp. 3298-3305, 2010.

[30] F. Wang and C. Liu, "Synchronization of unified chaotic system based on passive control," Physica D. Nonlinear Phenomena, vol. 225, no. 1, pp. 55-60, 2007.

[31] W. Xiang-Jun, L. Jing-Sen, and C. Guan-Rong, "Chaos synchronization of Rikitake chaotic attractor using the passive control technique," Nonlinear Dynamics. An International Journal of Nonlinear Dynamics and Chaos in Engineering Systems, vol. 53, no. 1-2, pp. 45-53, 2008.

[32] X. Zhou, L. Xiong, W. Cai, and X. Cai, "Adaptive synchronization and antisynchronization of a hyperchaotic complex Chen system with unknown parameters based on passive control," Journal of Applied Mathematics, vol. 2013, Article ID 845253, 8 pages, 2013.

[33] C. J. Wu, Y. B. Zhang, and N. N. Yang, "The synchronization of a fractional order hyperchaotic system based on passive control," Chinese Physics B, vol. 20, no. 6, Article ID 060505, 2011.

[34] D. Matignon, "Stability results for fractional differential equations with applications to control processing," in Proceedings of the Computational Engineering in Systems and Application Multiconference, pp. 963-968, 1996.

[35] F. Q. Wang and C. X. Liu, "Study on the critical chaotic system with fractional order and circuit experiment," Acta Physica Sinica, vol. 55, no. 8, pp. 3922-3927, 2006. 


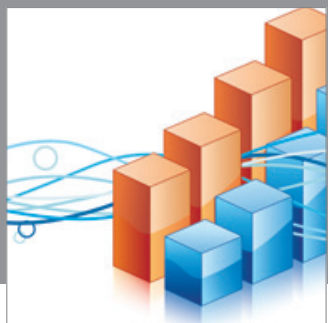

Advances in

Operations Research

mansans

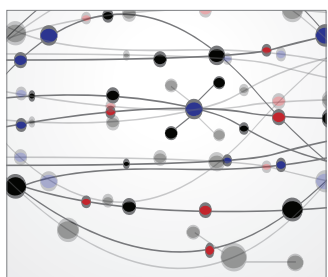

The Scientific World Journal
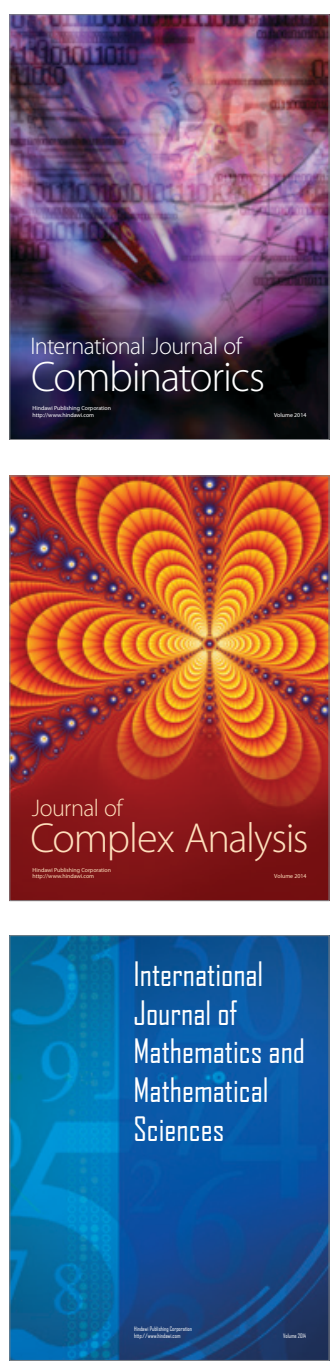
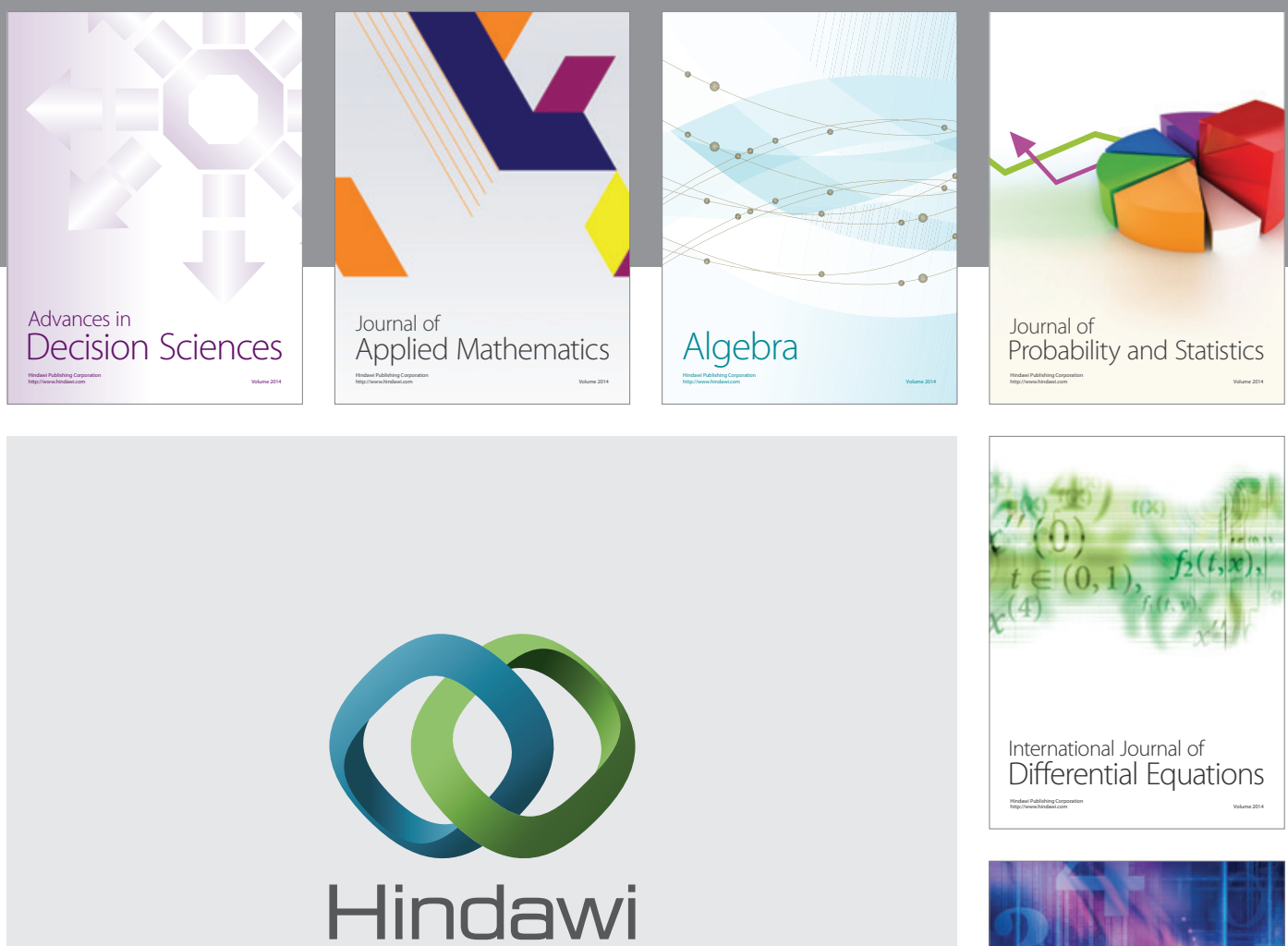

Submit your manuscripts at http://www.hindawi.com
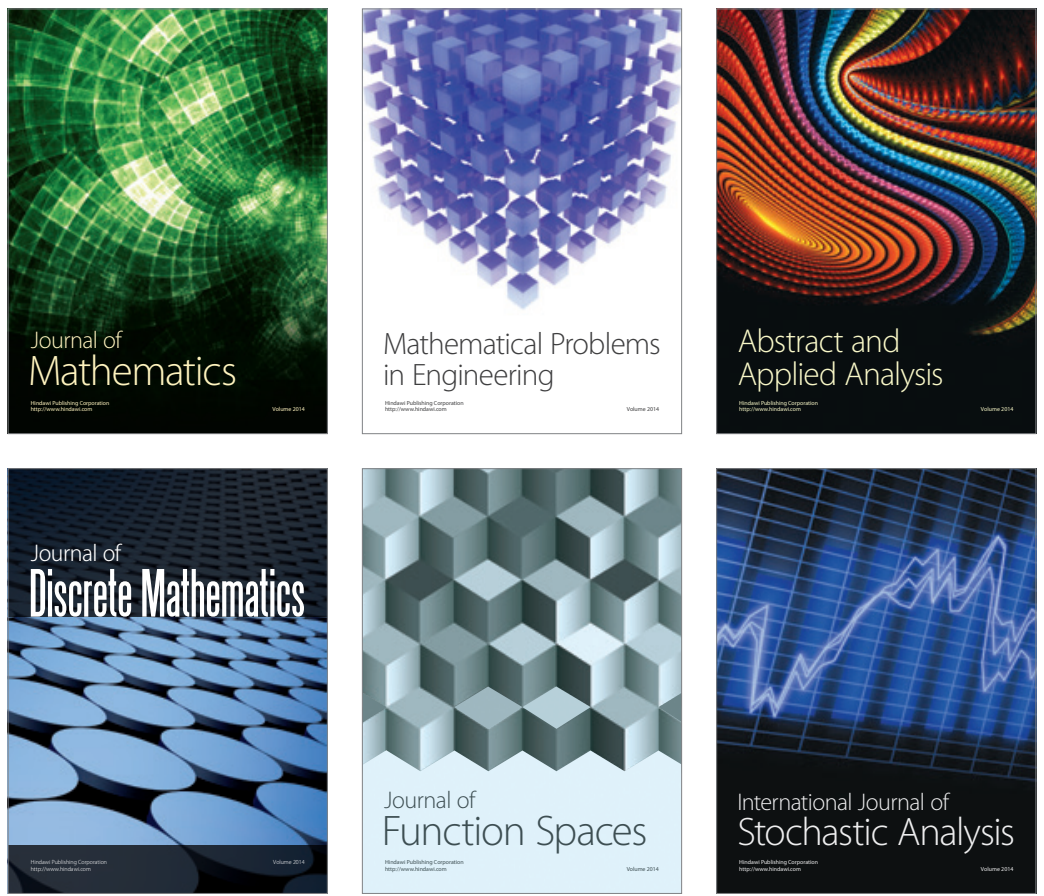

Journal of

Function Spaces

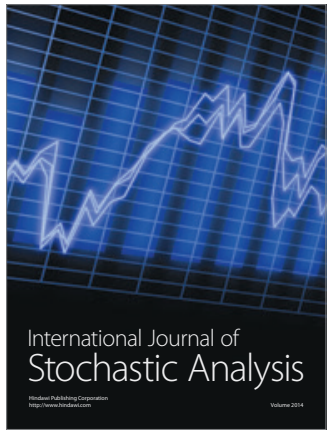

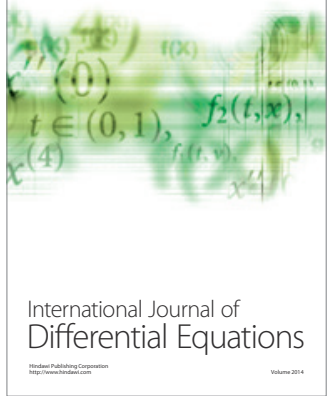
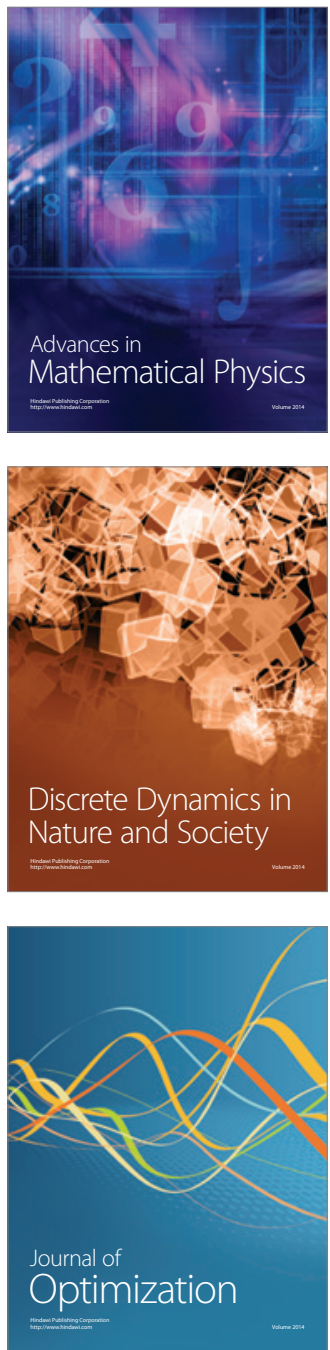